\title{
Enchantement, distraction et autoreprésentation : grottes artificielles entre l'Italie et la France pendant la Renaissance
}

\section{Sabine Frommel}

\section{(2) OpenEdition}

1 Journals

Édition électronique

URL : http://journals.openedition.org/artefact/5272

DOI : 10.4000/artefact.5272

ISSN : 2606-9245

Éditeur :

Association Artefact. Techniques histoire et sciences humaines, Presses universitaires du Midi

Édition imprimée

Date de publication : 15 juillet 2020

Pagination : 19-37

ISBN : 978-2-8107-0691-4

ISSN : 2273-0753

Référence électronique

Sabine Frommel, «Enchantement, distraction et autoreprésentation : grottes artificielles entre l'Italie et la France pendant la Renaissance », Artefact [En ligne], 12 | 2020, mis en ligne le 21 décembre 2020, consulté le 23 décembre 2020. URL : http://journals.openedition.org/artefact/5272 ; DOI : https:// doi.org/10.4000/artefact.5272

\section{(c) $(1) \Theta$}

Artefact, Techniques, histoire et sciences humaines est mise à disposition selon les termes de la Licence Creative Commons Attribution - Pas d'Utilisation Commerciale - Pas de Modification 4.0 International. 


\section{Enchantement, distraction et autoreprésentation : grottes artificielles entre l'Italie et la France pendant la Renaissance}

\section{Sabine Frommel}

$\mathrm{L}$ es fouilles conduites au nymphée de Noisy, qui continuent à faire émerger des témoignages pertinents, ont stimulé un nouveau débat européen autour des grottes artificielles à la Renaissance et des réseaux de migration auxquels elles doivent leur apparition dans plusieurs pays d'Europe. Un premier colloque gravitant autour de la fameuse grotta degli Animali a eu lieu à la Villa Médicis de Castello le 22 février 2018 (actes publiés dans un numéro spécial de la revue Opus Incertum, nuova seria, anno IV, 2018), suivi par des séminaires à Noisy-le-Roi le 2 novembre 2018, puis à l'université de Gênes les 9-10 décembre 2019. Ces manifestations ont révélé un riche panorama d'occurrences et de réflexions, invitant à enquêter sur les filiations, les persistances et les innovations de cette catégorie architecturale. Le développement de celle-ci, comme d'autres typologies de la Renaissance, a évolué sous le signe d'une assimilation de modèles provenant de l'Antiquité romaine, d'une recherche de langages éloquents d'autoreprésentation, d'un penchant pour l'hédonisme tendant à façonner les espaces d'une atmosphère mystérieuse et édifiante, rehaussée d'effet d'émerveillement. Dans de nombreux cas, il s'agit de véritables œuvres d'art totales qui font dialoguer architecture, sculpture, peinture, installations

95 Sabine Frommel, «Enchantement, distraction et autoreprésentation : grottes artificielles entre l'Italie et la France pendant la Renaissance », Artefact, 12, 2020, p. 19-37. 
hydrauliques, pièces de collection et jardin. La décoration profita beaucoup des découvertes et études des monuments romains, notamment la Domus aurea de Néron et ses grotesques. Outre leur irrésistible impact esthétique, rehaussé d'une force quasi magique, ces grottes reflètent souvent un intérêt scientifique pour les minéraux, leurs différentes formations et réactions, et même dans certains cas pour la végétation et les animaux, avec une attention particulière accordée aux espèces exotiques. Ces créations suscitèrent l'attention des poètes et écrivains qui, par de merveilleuses descriptions et interprétations, contribuèrent à leur diffusion et à leur fortune. L'objectif de ce texte n'est pas de brosser l'histoire des grottes artificielles, qui a déjà été écrite maintes fois, mais d'évoquer quelques épisodes significatifs, susceptibles de lever le voile sur certains dynamismes de transmission entre la France et l'Italie'.

\section{La métamorphose du concept de la grotte}

$\mathrm{Au}$ Quattrocento, le thème de la grotte est fréquent, notamment dans la peinture religieuse, mais aussi dans les scénographies de théâtre ${ }^{2}$. Des thèmes comme la naissance de Jésus dans une grotte (tiré de l'Évangile du Pseudo Matthieu), l'adoration des bergers ou des rois mages, la rencontre entre le Christ et saint Jean-Baptiste dans une caverne - épisode très présent dans l'iconographie florentine - jusqu'à la Vierge assise dans une excavation rocheuse, stimulèrent l'imagination des artistes, notamment Andrea Mantegna, Jacopo Bellini ou Léonard de Vinci. Une scénographie dessinée par ce dernier vers 1506-1508 (Codex Arundel f. 231v et 224r), d'après une mise en scène qu'il avait lui-même élaborée à Mantoue en 1490 pour une représentation d'Orphée, illustre le lien entre théâtre et grotte $^{3}$. Représentation de l'enfer, la grotte était comme creusée dans une montagne et, en fonction des épisodes de la narration, elle devait successivement s'ouvrir et se fermer.

1. Les grottes de la Renaissance ont bénéficié des nombreuses études auxquelles nous renvoyons le lecteur, par exemple Morel, 1998 ; Cazzato, 2001 ; Cazzato, 2002 ; Romana Liserre, 2008.

2. Pochat, 1990 , p. 227-228.

3. Taglialagamba, 2019, p. 94. 
Sur le plan architectural, la grotte s'inscrit dans une longue tradition qui remonte à l'Antiquité romaine : dans ce genre de cavernes, on célébrait le culte de certaines divinités, notamment des nymphes, filles de Zeus. Selon le mythe, ces personnifications des activités créatives et productives de la nature prennent naissance avec les eaux de pluie, recueillies ensuite sous terre pour enfin en sortir sous forme de sources. La Grotte bleue de l'île de Capri, celle de Tibère à Sperlonga - une caverne naturelle agrémentée de groupes sculptés monumentaux - ou encore celles d'Égérie à Rome et de la Villa Adriana à Tivoli donnent une idée des spécificités ainsi que de la variété de ces espaces au caractère magique. Ces grottes ou nymphées - les seconds présentant un ordre architectural plus raffiné - furent découverts par des artistes et des humanistes passionnés par l'héritage classique. Outre des témoignages monumentaux, conservés souvent sous forme fragmentaire, les sources écrites relatives à ce genre stimulèrent l'appropriation de telles constructions au sein des demeures de la Renaissance. Dans son traité De Re aedificatoria (IX, 4), conçu en 1452 et publié en 1485, Leon Battista Alberti décrit une grotte dans le cadre d'une habitation privée. Il préconise que la paroi soit revêtue d'une couche de spuma de travertin (littéralement « mousse » ou « écume » de travertin), qu'Ovide appelle pomice viva (pierre ponce), dont la surface est rugueuse et sobre. Le lichen peut être imité grâce à l'ajout de certaines couleurs, ce qui en exalte l'apparence naturelle. Alberti apprécie le revêtement fait de coquillages et d'huîtres arrangés dans un sens ou dans l'autre, de manière à varier gracieusement les couleurs. Au sein de cet agencement, l'eau, activée par différents mécanismes, coule, jaillit ou goutte. Dans l'ambiance d'un palais ou d'une villa, ce genre de cavité favorisait le lien direct entre l'homme et la nature et invitait à des expériences particulières en dehors du cérémonial souvent contraignant. La représentation de thèmes mythologiques, l'un des sujets chers à l'humanisme, et la connotation religieuse évoquant le lieu de naissance du Christ ne cessèrent de motiver la mise au point de nouvelles formules artistiques. Dès le début $\mathrm{du} \mathrm{XvI}^{\mathrm{e}}$ siècle, la typologie connut un développement qui s'accompagna de nombreuses déclinaisons - bains, thermes et autres installations hydrauliques - témoignant d'une exégèse de plus en plus poussée du traité de Vitruve. Article d'exportation prestigieux, la grotte artificielle telle qu'elle a été décrite par Alberti bénéficia d'une longue fortune et se transforma au contact de différents milieux culturels, dans presque toute l'Europe. 


\section{Quelques étapes dans un important mouvement de migration en Italie}

Dès le début du pontificat de Jules II en 1503, alors que les architectes s'attachaient à une interprétation de plus en plus savante des modèles provenant de l'Antiquité romaine et de la théorie vitruvienne, l'intérêt pour la typologie de la grotte artificielle s'intensifia aussi. Dans la cour du Belvédère, sur la terrasse intermédiaire, Bramante creusa un nymphée dont l'entrée était flanquée par des rampes divergentes conduisant au niveau supérieur, le cortile della Pigna. S'ouvrant par un arc de triomphe, son intérieur était doté d'une abside dont les niches d'un format élancé rappellent l'organisation des espaces périphériques dans le projet pour la basilique Saint-Pierre du même architecte (GDSU 1)4. La calotte était garnie d'une coquille et une partie de la paroi était revêtue de pierre volcanique. Une des niches accueillait le bassin d'une fontaine qui aurait agrémenté cette caverne servant par ailleurs aux représentations théâtrales. Dans la villa à l'antique de Genazzano, appartenant au cardinal Colonna et située en contrebas de son palais, le nymphée est creusé dans la colline située dans l'axe de la construction ${ }^{5}$. L'exèdre, munie de niches tantôt semicirculaires, tantôt rectangulaires, se poursuit au niveau de la travée centrale par une petite excroissance dotée d'un bassin où l'eau devait couler depuis une cavité du mur. En 1518, dans son projet pour la Villa Madame (GDSU 273), voulu par Léon X, Raphaël s'inspire de cette construction originale et insère un nymphée semi-circulaire au milieu du mur postérieur de la cour rectangulaire ${ }^{6}$. La réalisation, issue d'une collaboration avec Antonio da Sangallo le Jeune, se limite à une évocation : une niche revêtue de coquillages fut percée dans un mur de soutènement de la colline. L'eau jaillissait de la trompe d'un éléphant. À la Farnésine, sur les rives du Tibre, Baldassarre Peruzzi construisit pour le banquier Agostino Chigi une grotte artificielle, formée d'une substruction surmontée par une loggia qui communiquait avec le jardin 7 . Il apparait que la disposition renvoie au nymphée de la fameuse Villa de Poggioreale près de Naples, exécuté

\footnotetext{
4. C. L. Frommel, 1998, p. 35-37.

5. C. L. Frommel 2003a, p. 218.

6. Ibid., p. 233-235.

7. C. L. Frommel 2003b, p. 22.
} 
en 1489, dont Peruzzi avait effectué un relevé (GDSU 363Ar) ${ }^{8}$. Ses excellentes connaissances de l'architecture de l'Antiquité romaine, documentée par de nombreux relevés, s'y expriment à merveille. Selon le panégyrique Suburbanum Augustini Chisii de Blosio Palladio (vers 1511), on pouvait s'y promener en bateau pour observer les nymphes et les poissons, alors que les lieux offraient une belle vue vers la colline du Janicule et sur la ville avec les monuments antiques. L'humaniste la qualifie de lieu où les dieux de l'Olympe auraient aimé se réunir et se baigner. Cette grotte servit aussi pour des banquets, comme celui, fameux, donné pour Léon X à l'été 1518. Ces créations constituèrent des références tout au long du XVI ${ }^{\mathrm{e}}$ siècle.

Dans le projet d'Antonio da Sangallo pour la villa du cardinal del Monte, dessiné vers 1525-1526 sur le terrain de la future Villa Giulia, une succession de cavernes alternativement circulaires et octogonales creusées dans le tuf entoure une partie du jardin (GDSU 842Ar) et tire habilement profit des ressources naturelles ${ }^{9}$. À la Villa imperiale à Pesaro, vers 1531, Girolamo Genga réalisa pour Francesco Maria della Rovere une grotte située au centre d'un corps de bâtiment autonome. Lartiste, qui avait profité du climat effervescent de Rome en 1518-1520 dans la bottega de Raphaël, renoua pour ce chantier avec le nymphée de Genazzano. Revêtue de pierre ponce rugueuse, la grotte de Pesaro prend l'apparence d'une caverne naturelle, éclairée par un puits de lumière. Elle est flanquée symétriquement de bains de format carré dotés de niches et communique avec un long bassin, creusé dans un rocher existant du côté postérieur ${ }^{10}$. Cette association atteste un caractère inédit, tout comme la façade monumentale vers la cour, ennoblie, de part et d'autre de l'arcade centrale, de pilastres ioniques d'un ordre colossal flanquant des niches superposées.

Au tournant de la troisième décennie du $\mathrm{XvI}^{\mathrm{e}}$ siècle, l'identification de la grotte d'Égérie ( $\mathrm{I}^{\mathrm{e}}$ siècle de notre ère), située sur la Via Appia, à l'extérieur de la porte Saint-Sébastien de Rome, initia une nouvelle phase de sa fortune iconographique. Son plan fut relevé par Antonio da Sangallo le Jeune (GDSU 1223Ar) et Sallustio Peruzzi (GDSU 655Ar, 689Ar), ce dernier s'appuyant probablement sur des dessins de son père Baldassarre ${ }^{11}$. À l'occasion de l'entrée triomphale de Charles Quint en 1536, un banquet

8. Sur ce nymphée, voir Modesti, 2014, p. 90.

9. S. Frommel, 2002, p. 20-24.

10. Pour une présentation synthétique de la villa, voir Fiore, 2002, p. 446-452.

11. Castellini, 2018, p. 36-38. 
fut organisé à cet endroit. Giovanni Gaddi, prélat florentin protégé des Médicis, supervisa cet événement sur le plan administratif ${ }^{12}$, alors qu'Antonio da Sangallo le Jeune développa le projet architectural en se servant des dessins de Peruzzi, mort cette année-làa ${ }^{13}$.

Gaddi fit lui-même construire une grotte au bord du Tibre, malheureusement disparue. Elle est cependant décrite dans une lettre de 1538 envoyée à Giovanni Guidiccioni par Annibal Caro, poète entré au service du prélat en $1525^{14}$. Tout porte à croire que cette grotte reflète une multiplicité d'influences. Le palais de Gaddi à Rome, construit par Jacopo Sansovino dans le Rione Ponte, fut un foyer pour un cercle d'humanistes passionnés par les études vitruviennes (il est à la base de l'Accademia vitruviana fondée en 1542), attirant aussi dans son élégante demeure des artistes, des écrivains et d'illustres personnages de la vie culturelle comme Benvenuto Cellini, Pietro Aretino, Benedetto Varchi et Niccolò Tribolo. D'après cette lettre et un dessin conservé à Oxford se référant sans doute à cette construction, le visiteur était accueilli au fond d'une pergola par un mur fait de blocs de tuf noir et spongieux placés de manière irrégulière con certo ordine disordinato $^{15}$. Une porte conduisait à un groupe de pièces dont les murs et les voûtes étaient également couverts d'un assemblage irrégulier du même matériau. Des pierres pendantes, imitant des stalactites, ainsi qu'une niche en forme de rocher creusé, accueillant un bassin garni de têtes de lion, évoquait une grotte naturelle. L'eau était présente à différents endroits, aux angles sous forme de fontaines, dans des pelaghetti (petits plans d'eau) et tombait à la manière d'une pluie depuis la voûte percée de trous. Un décor de coraux, de rochers, de perles, de nacre, d'escargots et de plantes aquatiques ornait la paroi tandis que la calotte était revêtue d'un dépôt blanc évoquant du givre. Des statues en marbre à l'effigie des fleuves ennoblissaient cet intérieur spectaculaire dont la variété de l'épiderme, des reliefs et des sons durent frapper le visiteur qui ne pouvait rester insensible à cette force évocatrice. En 1538, au moment où Caro rédigeait sa lettre, l'artiste portugais Francisco da Holanda visitait la Ville éternelle et fut également impressionné par des grottes. Il effectua un relevé de celle d'Égérie

12. Dans sa jeunesse, Gaddi fut proche de Julien de Médicis duc de Nemours et fit concevoir des scénographies de théâtre par Jacopo Sansovino, Andrea del Sarto et Giovan Francesco Rustici.

13. Quant à cet événement, voir note 28 dans Ferretti et Lo Re 2018, p. 17.

14. Caro, 1957-1961, vol. I, p. 105-109. Sur Giovanni Gaddi voir Arighi, 1998.

15. Elam, 2013, p. 446-462. 
(Fig. 1) et dessina une composition non identifiée, marquée par deux figures symbolisant la fécondité (Diane d'Éphèse), érigées dans un bassin à l'intérieur d'une grotte artificielle faite de blocs de pierre grossièrement taillés et arrangés.

Fig. 1. - Francisco de Holanda (1517-1585), Os desenhos das antigualhas

Madrid, Bibliothèque royale
du monastère de l'Escorial, 28-I-
20, fol. 33v.

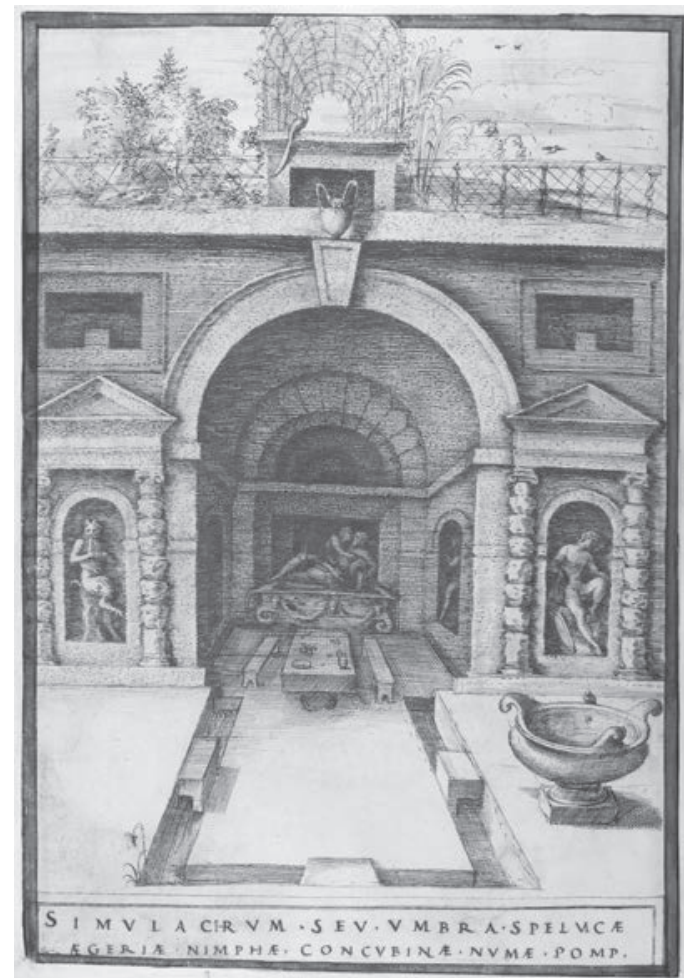

Lorsqu'au printemps 1538 et à l'été 1539, Niccolò Tribolo rencontra Annibal Caro à Rome, il aurait pu s'informer à propos de la grotte de Giovanni Gaddi et peut-être même la visiter ${ }^{16}$. En tout cas, cette expérience se reflète dans la grotta degli animali de la villa Médicis à Castello, liée à un vaste projet d'aménagement de jardin commandé par Cosme ${ }^{\text {er }}$ de Médicis en 1537, pour lequel Tribolo collabora avec l'ingénieur hydraulique Davide Fortini ${ }^{17}$. Le lien avec Rome est d'ailleurs plus complexe, puisque le plan renvoie clairement à la grotte d'Égérie et au mythe de Numa Pompilius. La figure de cette nymphe allait remplir un rôle important dans les stratégies

16. Ferretti et Lo Re, 2018, p. 19.

17. Plus tard interviendront Giorgio Vasari et Bartolomeo Ammannati et enfin Bernardo Buontalenti et Raffaello di Pagno. Voir les articles relatifs à cette grotte dans Ferretti, Frommel, Giannotti, Mozzo, 2018. 
encomiastiques des Médicis au cours des années 1540 et 1550 . Tout cela atteste que, malgré des tensions entre Cosme ${ }^{\text {er }}$ de Médicis et le pape Paul III Farnèse, les liens étroits entre Rome et Florence, qui s'étaient intensifiés pendant les pontificats de Léon X et Clément VII, connurent un développement fertile. Dans le même temps, cette grotte spectaculaire marque une nouvelle étape dans l'évolution décorative et symbolique du genre, dans laquelle culminent les recherches menées dans ce domaine dans la capitale toscane ${ }^{18}$. La culture napolitaine d'Éléonore de Tolède, épouse de Cosme I ${ }^{\text {er }}$ de Médicis à partir de 1539 et commanditaire passionnée, eut certainement une influence sur cet essor.

Dès le début des années 1540, la grotte artificielle se diffusa largement dans les cercles les plus cultivés, non seulement en Italie, mais dans toute l'Europe. À Gênes, un mouvement important fut initié avec la grotte située dans le jardin du palais d'Andrea Doria, projetée par Galeazzo Alessi qui s'était installé dans la ville à la fin des années 1540 (Fig. I, cahier couleur). La construction s'élève sur un plan octogonal couronné d'une coupole originellement dotée d'un puits de lumière ${ }^{19}$. Les arcades qui bordent l'espace centré, renvoyant aux thermes romains, sont soutenues par des piliers sur lesquels sont plaqués des ordres anthropomorphes. Une excroissance située dans l'axe de l'entrée, dépourvue d'éclairage et ornée de stalactites, attire le regard dans la profondeur. Tant la surface des arcades aveugles, disposées en retrait, que les voûtains de la coupole sont couverts de coquillages, coraux et cristaux avec lesquels sont façonnés des figures mythologiques, jusqu'au moindre détail du drapé, ainsi que des architectures feintes. Dans cette grotte, l'eau constitue à nouveau un élément clef : elle devait couler, goutter et jaillir sous diverses formes et avec différentes intensités. Cette grotte artificielle doit beaucoup à l'expérience d'Alessi à Rome entre 1536 et 1542, où il avait bénéficié de la protection du cardinal Ascanio Parisani, proche du cardinal Del Monte et des Farnèse. Ainsi, il a sans doute profité de contacts avec l'élite culturelle de la cité pontificale, notamment avec l'Accademia Vitruviana déjà citée, de laquelle faisait aussi partie Annibal

18. Plusieurs articles gravitent autour de cette grotte dans Ferretti, Frommel, Giannotti, Mozzo, 2018.

19. Voir Magnani, 1984. Nous attendons la publication des actes de colloque Grotte artificiali di giardino. Genova nel panorama europeo, colloque international organisé sous la dir. de Sabine Frommel et Lauro Magnani, Università degli Studi di Genova, DIRAAS (Dipartimento di italianistica, romanistica, antichistica, arti e spettacoli), Palazzo Balbi Cattaneo, Via Balbi 2, Villa Pavese, Sampierdarena, Via Nicolà Daste 9, Gênes, 9-10 décembre 2019. 
Caro. À Gênes, la grotte d'Andrea Doria instaura une tradition prodigieuse qui se poursuivit jusqu'au XIX ${ }^{\mathrm{e}}$ siècle et produisit des chefs-d'œuvre toujours plus raffinés.

\section{L'adoption de la grotte artificielle en France : un parcours prodigieux}

À la cour de Fontainebleau, l'arrivée de Rosso Fiorentino (1530), de Francesco Primaticcio (1531) et de Sebastiano Serlio (1541) a provoqué certaines métamorphoses, notamment dans le domaine de la décoration des intérieurs et des arts figuratifs. Dans un pavillon existant au sud-ouest du château, Primatice réalisa à partir de 1543 la grotte des Pins, orientée vers le jardin du même nom ${ }^{20}$. Sa façade témoigne d'une réflexion inédite (Fig. II, cahier couleur) : trois arcades rustiques faites de bossages irréguliers, couronnées par des frontons triangulaires grossièrement dessinés, font que la construction semble inachevée, voire sur le point de s'écrouler. La composition renvoie à la loggia de la Rustica (1538-1539) de Giulio Romano dans la cour du palais ducal de Mantoue, que Primatice aurait pu visiter lors de son séjour italien, interrompu de manière inattendue par la mort de Rosso en novembre 1540. Sur les piliers sont adossés des corps humains, également en bossages, dont les bras semblent attachés derrière l'imposte des arcades. Dans un effort farouche et pathétique, ils tentent en vain de se libérer et leur expression montre la souffrance de ces géants pétrifiés qui ont l'air d'échanger entre eux à propos de leur pénible destin. Cette mise en scène évoque autant l'influence de la fresque de la chute des géants de Giulio Romano à Mantoue que les esclaves de Michel-Ange pour le tombeau de Jules II. L'espace intérieur, un rectangle de $7 \mathrm{~m}$ sur 4,5 m environ, est couvert d'une voûte en berceau surbaissée, subdivisée en trente-neuf compartiments délimités par de petits blocs d'aragonite et par trois fresques montrant Junon et Minerve sous un édicule de bois représenté par un raccourci perspectif, probablement attribuable à Jacopo Barozzi da Vignola. Le programme iconographique semble lié à la galerie d'Ulysse au premier étage, décorée par le même Primatice : on y retrouve des épisodes de l'Odyssée, dont Junon et Minerve sont des protagonistes,

20. Parmi la littérature scientifique abondante concernant cette grotte, voir S. Frommel 2010, p. $87-98$. 
et le thème de la pétrification, qui marque une des étapes de la souffrance du héros. Jusqu'à présent, on n'a pas pu mettre en évidence la présence de l'eau dans cette grotte, ce qui lui confère une place particulière dans cette filiation.

Le thème de la grotte dotée d'une façade rustique fascina aussi Serlio dans son projet de Padiglione in costume di Franza, conçu pendant les mêmes années pour le grand jardin du château de Fontainebleau ${ }^{21}$. En tant qu'édifice autonome, regroupant une grotte, des bains et des pièces d'habitation, ce pavillon renvoie sur le plan typologique au nymphée de Genazzano et à celui de la Villa imperiale à Pesaro. Pourtant, le fait que le Bolonais cherche à associer ces fonctions dans un édifice centré qui forme un volume libre s'avère inédit. Le rez-de-chaussée est articulé a costume di grotta, c'est-àdire que l'espace circulaire au milieu est éclairé parcimonieusement par quelques ouvertures percées dans le socle du belvédère du premier étage. Cette espèce de caverne, dépourvue de revêtement aux murs, est ceinte de niches, tantôt munies de portes, tantôt destinées à accueillir des statues, alors que les autres pièces sont régulièrement groupées autour. Le projet demeura sur le papier, mais l'idée d'une grotte sous forme autonome bénéficia d'une grande fortune en France, comme le montrent celles des châteaux de Meudon et de Noisy.

La grotte du château de Meudon, une maison de plaisance située à 120 mètres du château, a été dessinée par Primatice en 1552 selon des idées du commanditaire, le cardinal de Lorraine - « certaines petites invantions que je y ait pensées ${ }^{22}$ ". L'édifice spectaculaire fut loué par Giorgio Vasari, généralement indifférent à l'architecture française, comme le palazzo della grotta. Rien ne reste de cette œuvre d'art totale qui faisait dialoguer architecture, sculpture et peinture et qui passionna aussi un poète comme Ronsard. Les gravures de Jean Marot attestent que la construction était habilement intégrée dans un vaste jardin en pente, organisé par une succession de terrasses qui rappellent la cour du Belvédère de Bramante au Vatican ou, plus tard, la Villa Médicis de Castello ${ }^{23}$. Des cryptoportiques et des jeux de rampes divergentes créaient un parcours scénographique, mais contrairement aux modèles italiens, la grotte était insérée dans un pavillon

21. Voir S. Frommel 2002a, p. 255-265 ; S. Frommel 2017, p. 66-88 (en particulier p. 66-69).

22. S. Frommel 2010, p. 119.

23. Ibid., p. 118-131. 
dont le toit pyramidal élevé couronnait deux étages et une mezzanine, abritant des espaces privés, représentatifs et des collections. Deux pavillons plus bas, situés aux extrémités, complétaient un rythme tripartite, centré sur la grotte placée au cour de l'ensemble. À l'instar de la grotte des Pins, la façade était faite de bossages rustiques, mais traduits ici dans un ordre prestigieux marqué par un avant-corps muni de colonnes toscanes engagées encadrant l'arcade centrale. La grotte formait un espace rectangulaire de 9,54 m sur 6,58 m doté de niches tantôt semi-circulaires, tantôt rectangulaires où devaient prendre place des statues ${ }^{24}$. Couvert par une voûte surbaissée, cet espace était agrémenté de fontaines, alors que les parois étaient revêtues de coquillages, de coraux et autres éléments marins, ainsi que de figures en stuc exécutées par le peintre Dominique Florentin. À en croire Bernard Palissy, la terre cuite sculptée et émaillée a également été utilisée pour évoquer des morceaux de roche ${ }^{25}$. Les compartiments de la voûte étaient ornés d'un cycle mythologique. La grotte de Meudon était dédiée à Henri II et aux muses, qui président aux arts libéraux, en s'inspirant d'un concept de Pline le Jeune. Ce décor rappelle la description d'Annibal Caro et il est possible que le cardinal de Lorraine ait vu la grotte de Gaddi pendant son séjour à Rome à l'occasion du conclave de 1549-1550. Le fait qu'en 1559 Catherine de Médicis nommât Primatice "surintendant des bastiments de la couronne » laisse entendre qu'elle dut aussi apprécier cette construction spectaculaire, qui constituait alors la référence principale du Bolonais dans le domaine monumental.

La tradition de la grotte comme foyer d'un édifice isolé culmine dans celle du château de Noisy, qu'Albert Gondi, duc de Retz et maréchal de France, avait fait réaliser en $1582^{26}$. Ce protégé de Catherine de Médicis reçut d'ailleurs la visite de la souveraine en octobre 1579. On note une double influence, d'une part florentine et d'autre part provenant du château de Meudon. L'invitation de qualche garzone de Pratolino, dont on ignore l'identité et la réponse à la sollicitation, atteste que le commanditaire souhaitait que l'on s'appuie sur une technique florentine ${ }^{27}$. Une connaissance par Gondi du chantier médicéen, lancé en 1568 par le grand-duc François I ${ }^{\text {er }}$ sur un projet de Bernardo Buontalenti, semble probable étant donné qu'un

24. Ibid., p. 130.

25. Ibid., p. 129

26. Voir l'article de Bruno Bentz dans ce volume avec les références bibliographiques.

27. Voir l'article de Marco Calafati dans ce volume. 
de ses parents du nom d'Alfonso en était administrateur des comptes ${ }^{28}$. S'inscrivant dans un rectangle d'environ $20 \mathrm{~m}$ sur $17 \mathrm{~m}$, la grotte est plus grande et d'une organisation plus complexe que celle de Meudon, cette dernière consistant en un seul espace de format rectangulaire creusé de niches (Fig. 2). Cette configuration n'affiche pas seulement l'ambition du commanditaire d'éclipser ce modèle prestigieux, mais aussi un programme fonctionnel plus riche et varié dont nous ignorons les détails. Manquent cependant les pavillons latéraux et les montées théâtrales et un seul étage surmonte le nymphée. Par un portique doté de quatre colonnes rustiques, on entrait dans un espace central formant un octogone, marqué par de profondes niches placées diagonalement. Dans les axes, des espaces de plan tréflé - des carrés également dotés de trois larges niches - se greffaient sur le corps central. Selon la gravure de Marot, ces structures périphériques étaient couronnées de lanternons qui sortaient de terre. Le plan évoque des bâtiments sacrés à plan centré d'origine italienne - on note des analogies avec Santa Maria della Consolazione à Todi -, tandis que le décor, consistant en rocailles et coquillages, était encore plus prodigieux qu’à Meudon et dégageait le faste d'un espace de représentation. L'apparence naturelle et parfois rustique cède ici la place, comme les gravures de Marot semblent l'affirmer, à un agencement architectural somptueux. Les fouilles récentes ont révélé des détails du décor et du système hydraulique ainsi que les différentes installations de l'eau coulant doucement, jaillissant ou se répandant avec vigueur.

Nous avons essayé de présenter les principales lignes de développement parmi le vaste processus de migration des grottes artificielles, mais celles-ci témoignent en réalité d'une extraordinaire variété. Pour sa grotte aux Tuileries, Catherine de Médicis chargea Bernard Palissy en 1570 d'une réalisation en céramique, imitant des formes et des espèces naturelles ${ }^{29}$. Si la reine privilégia aussi la forme isolée pour cette construction, d'autres furent insérées dans les demeures comme à la Bastie d'Urfé, dont le commanditaire Claude d'Urfé fut ambassadeur de François I ${ }^{\text {er }}$ au Concile de Trente, puis d'Henri II auprès du pape Paul III en 1548. Sous l'influence de ces expériences italiennes, il compléta entre 1548 et 1558 l'aménagement de son château avec une chapelle et une grotte. Dans cette dernière, de

28. Calafati, 2018, p. 136.

29. Anne de Montmorency, connétable de France, avait également commandé une telle construction au même artiste (Ferdinand, 2019, p. 110-114). 


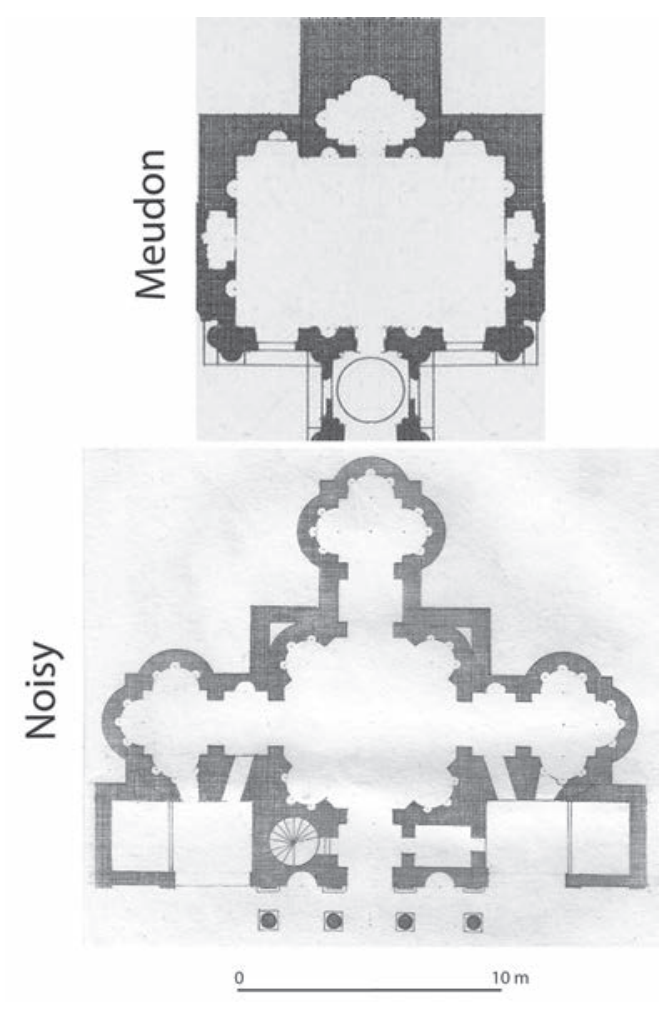

Fig. 2. - Comparaison des plans des grottes de Meudon et de Noisy, gravées par Marot vers 1650

Coll. particulières.

Montage et cliché Sabine Frommel

nombreux détails évoquent la grotte d'Andrea Doria à Gênes : des termes et des figures en gaines qui s'adossent sur les piliers des arcades, des niches dotées de stalactites et ornées des coquillages ou de minéraux. Le couvrement s'éloigne en revanche des modèles italiens : un plancher en bois formant des caissons "à la française " est décoré par divers sables colorés ponctués de coquillages ${ }^{30}$. Étant donné que cette grotte forme l'atrium de la chapelle, son programme iconographique embrasse des motifs sacrés qui préludent à ceux de l'espace cultuel. Au château de Maulnes, construit à partir de 1566 dans le Tonnerrois par le duc et la duchesse d'Uzès, proches de la reine, un nymphée entouré de gradins à l'instar d'un amphithéâtre est inséré dans la partie orientée vers le jardin ${ }^{31}$.

30. Voir l'article de Jean-François Grange-Chavanis dans ce numéro.

31. Voir la représentation de Jacques Androuet du Cerceau dans Les plus excellents bastiments de France. 
Tout compte fait, en France les grottes et nymphées évoluèrent essentiellement dans l'ambiance de la cour et devinrent une composante presque indispensable de l'habitat princier et aristocratique. Les traits spécifiques de la vie sociale provoquèrent la transformation des modèles italiens, avec par exemple leur intégration dans un pavillon d'habitation autonome, obéissant à un programme fonctionnel complexe.

\section{La grotte artificielle : objet de dialogue, de contamination et de transmission}

Albert Gondi ne fut pas le seul à s'adresser à la cour des Médicis, car des lettres attestent que Guillaume V de Bavière, en réalisant après 1579 la Cour des grottes de sa résidence munichoise, sollicita aussi en Toscane la livraison de matériaux (coquilles, cristaux, ornements marins et mousse) ${ }^{32}$. Henri IV, en 1598-1599, se fit envoyer des matériaux de construction et des ornements naturels, d'origine terrestre ou marine, pour servir à la confection de décors de rocaille sur les chantiers de Saint-Germain-enLaye et Fontainebleau ${ }^{33}$. Une véritable compétition se déclencha autour des créations les plus originales et spectaculaires et cette vague de migration se profila par un réseau de princes ambitieux aux motivations hédonistes. Malgré l'authenticité des matériaux, les techniques locales faisaient que le relief de l'épiderme et les effets de scintillement des surfaces offraient une certaine diversité. Si en Italie le revêtement faisait partie de la substance murale, en France on l'appliquait après coup, par couches successives.

Les fonctions de tels espaces s'avèrent similaires dans les différents pays, revêtant souvent d'une dimension politique, comme le révèlent les blasons, emblèmes et allusions à la dynastie, ou encore les thèmes mythologiques liés à des programmes encomiastiques. Ces capricci participent, ou forment même le point culminant d'un parcours scénographique qui lie la demeure, le jardin, des terrasses et des rampes d'aspect théâtral. Le lieu de la grotte et son accessibilité au sein d'un système intégral dépendaient à la fois des ressources naturelles et de la mise en scène voulue par le commanditaire et ses artistes. Au Palazzo ducale del Giardino à Parme, réalisé en 1561 pour Octave Farnèse au milieu d'un parc, la grotte se situe au

32. Deutsch, 2018, p. 108.

33. Lurin, 2018, p. 116. 
centre du rez-de-chaussée, en face de l'entrée ; les appartements se groupent autour et au-dessus de cet espace ${ }^{34}$. Par cette option non conventionnelle, le prince s'écartait du palais Farnèse à Plaisance, résidence que sa femme Marguerite d'Autriche édifia en même temps pour rivaliser avec les plus novatrices et prestigieuses de la péninsule.

Dans plusieurs cas - la grotte d'Andrea Doria à Gênes et celle de Noisy -, on note des similitudes avec l'architecture sacrée, et de manière encore plus forte dans la grotta Pavese à Gênes (1594), desservie par un vestibule à l'instar d'une église ${ }^{35}$. Cela s'explique tout d'abord par le fait que, pendant la Renaissance, l'architecture, qu'elle soit privée, publique ou religieuse, renoue avec des prototypes antiques comme les thermes et les bains, typologies caractérisées par leur clarté géométrique. Le plan octogonal couronné d'une coupole à lanternon bénéficia d'une grande fortune et favorisa la perméabilité entre différents types de bâtiment. Le creusement de la substance murale par des niches, qui se combinait à merveille avec l'exposition d'œuvres d'art souvent rehaussée par des jeux d'eau comme toile de fond, renvoie également à l'héritage classique. Par ailleurs, la signification religieuse de la grotte comme lieu de la naissance du Christ a certainement persisté et invité à des déclinaisons et sublimations de motifs religieux.

En tout cas, contrairement aux espaces appartenant à l'enfilade officielle, la grotte artificielle offrait une plus grande liberté pour suivre un goût personnel en dehors des conventions et surprendre par de nouvelles formules, évocations et combinaisons - une liberté qui dans certains cas pouvait nuire à la qualité esthétique. Une place d'exception est accordée aux ordres anthropomorphes, qui exaltent l'illusion par leurs fonctions tantôt porteuses, tantôt pseudo-porteuses, et offrent une diversité dans les traits du visage, dont l'expression va de la souffrance et de la tristesse jusqu'au ridicule et au bizarre ${ }^{36}$.

Enfin, la fortune fulgurante de la grotte artificielle pendant l'époque moderne fut encouragée par de multiples instruments de transmission, à commencer par les visites de curieux, des artistes itinérants, des descriptions, des dessins, des gravures jusqu’à la littérature et la poésie. Difficile à éclaircir est la question des retombées en Italie de certains modèles

34. S. Frommel 2013, p. 159-162.

35. Magnani, 1984.

36. Sur les ordres anthropomorphes, voir S. Frommel 2018. 
transalpins passés au filtre du goût français. Après son retour dans la péninsule (vers 1549), le cardinal Hippolyte d'Este fit effectuer, au Quirinal et ensuite à la Villa d'Este à Tivoli, des aménagements parmi les plus spectaculaires de la Renaissance européenne en accordant un rôle particulier aux jeux d'eau, aux grottes artificielles et aux nymphées ${ }^{37}$. Ils fournirent un énorme creuset de modèles et de références en Europe, comme le montrent, à titre exemplaire, les dessins et gravures de la fontana del Bosco (Quirinal) (Fig. 3), à partir de l'architecte allemand Heinrich Schickhardt qui visita le jardin à la fin du $\mathrm{XvI}^{\mathrm{e}}$ siècle $^{38}$. On en sait trop peu sur les jardins somptueux du cardinal dotés d'installations hydrauliques à Chaalis et au Grand Ferrare à Fontainebleau pour pouvoir conclure sur l'éventuelle appropriation d'expériences menées dans son pays d'accueil. Même si dans le cas d'un genre si fragile et fugace comme la grotte artificielle de nombreux témoignages ont disparu ou sont conservés de manière fragmentaire, la question du va-et-vient des traditions et des acquisitions entre plusieurs pays est appelée à demeurer un pivot de nos enquêtes.

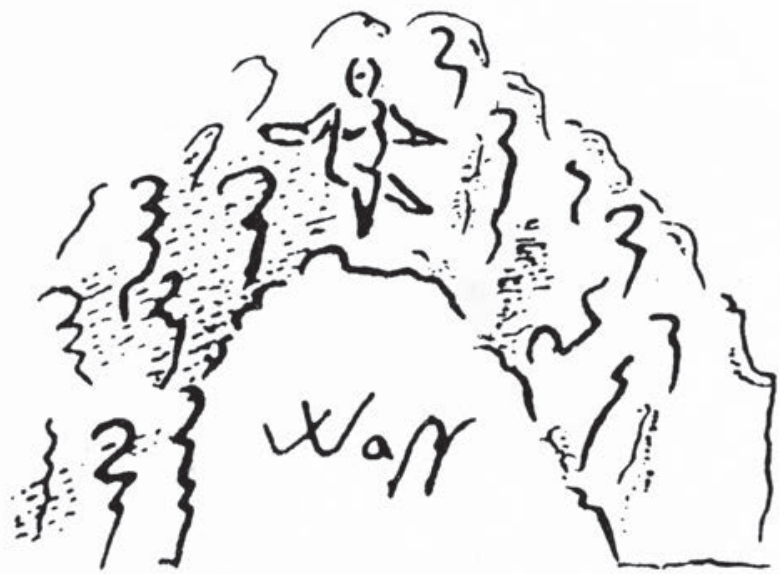

Fig. 3. - Heinrich Schickhardt (1558-1635), esquisse de la fontaine del Bosco, Stuttgart, Landesmuseum (cod. hist. QS 148b)

Cliché d’après Christian Hülsen, Römische Antikengärten des XVI. Jahrhunderts, Heidelberg, Carl Winters, 1917, fig. 84.

37. Quant aux jardins du Quirinal, voir C. L. Frommel 1999, p. 15-62. Sur la Villa d'Este à Tivoli, voir les contributions du volume Ippolito II d'Este. Cardinale, principe, mecenate, 2013.

38. C. L. Frommel, 1999, p. 39. 


\section{Bibliographie}

Arighi Vanna, "Giovanni Gaddi ", Dizionario Biografico degli Italiani, vol. 51, Istituto della Enciclopedia italiana, Rome, 1998.

Bentz Bruno, "Le décor de la grotte de Noisy : résultat des fouilles de 2017 ", dans Ferretti, Frommel, Giannotti et al (dir.), "Il Rinascimento delle grotte. Natura, arte e architettura fra Italia e Francia nel Cinquecento ", Opus Incertum, Nuova Serie, anno IV, 2018, p. 126-135.

Calafati Marco, "Acconciar grotti" alla villa di Pratolino e nei giardini del castello del Gondi a Noisy-le-Roi ", dans Ferretti, Frommel, Giannotti et al (dir.), "Il Rinascimento delle grotte. Natura, arte e architettura fra Italia e Francia nel Cinquecento ", Opus Incertum, Nuova Serie, anno IV, 2018, p. 136-141.

Caro Annibale (dir.), Lettere familiari, 3 vol., A. GreCo, Florence, 1957-1961.

CAstellini Marta, "Il ninfeo di Egeria (II sec. D. C.) e la grotta degli animali a Castello. Il ruolo del Modello antico attraverso l'analisi dei disegni del GDSU » dans Ferretti, Frommel, Giannotti, Mozzo (dir.), «Il Rinascimento delle grotte. Natura, arte e architettura fra Italia e Francia nel Cinquecento ", Opus Incertum, Nuova Serie, anno IV, 2018, p. 36-43.

Cazzato Vincenzo (dir.), Atlante delle grotte e dei ninfei in Italia: Italia settentrionale, Toscana, Lazio, Italia meridionale e Isole, Electa, Milan, 2001.

Cazzato Vincenzo (dir.), Atlante delle grotte e dei ninfei in Italia: Italia settentrionale, Umbria e Marche, Electa, Milan, 2002.

Cogotti Marina et Fiore Francesco Paolo, Ippolito II d'Este. Cardinale, principe, mecenate (actes du colloque a Villa d'Este à Tivoi, 13 mai 2013), De Luca, Rome, 2013.

Deutsch Kristina, "Grotte, bain et jardin : leur lien dans les châteaux des Wittelsbach pendant la Renaissance ", dans Ferretti, Frommel, Giannotti et al (dir.), "Il Rinascimento delle grotte. Natura, arte e architettura fra Italia e Francia nel Cinquecento ", Opus Incertum, Nuova Serie, anno IV, 2018, p. 2-111.

Elam Caroline, " "Con certo ordine disordinato". Images of Giovanni Gaddi's Grotto and other Renaissance foundations " dans IsRAëLs Machtelt, WaLdMAN Louis A. (dir.), Renaissance Studies in honor of Joseph Connors, t. I (Art History), The Harvard University Center for Italian Renaissance Studies, Florence, 2013, p. 446-464.

Ferdinand Juliette, Bernard Palissy. Artisan des réformes entre art, science et foi (European Identities and transcultural exchange. Studies in Art History), De Gruyter, Berlin/Boston, 2019. 
Ferretti Emanuela et Lo Re Salvatore, «Il ninfeo di Egeria sulla Via Aoppia e la grotta degli animali di Castello: Mito e architettura tra Roma e Firenze ", dans Ferretti, Frommel, Giannotti, Mozzo (dir.), « Il Rinascimento delle grotte. Natura, arte e architettura fra Italia e Francia nel Cinquecento ", Opus Incertum, Nuova Serie, anno IV, 2018, p. 14-23.

Ferretti Emanuela, Frommel Sabine, Giannotti Alessandra, Mozzo Marco (dir.), "Il Rinascimento delle grotte. Natura, arte e architettura fra Italia e Francia nel Cinquecento ", Opus Incertum, Nuova Serie, anno IV, 2018.

Fiore Francesco Paolo, "Urbino, Pesaro e Girolamo Genga ", dans Bruschi Arnaldo (dir.), Il Primo Cinquecento (Storia dell'Architettura Italiana), Electa, Milan, 2002, p. 446-455.

Frommel Christoph Luitpold, "I tre progetti bramanteschi per il cortile de Belvedere ", dans Winner, Andreae et Pietranegli (dir.), Il cortile de Statue. Der Statuenhof des Belvedere im Vatican, Mainz, 1998, p. 17-65.

Frommel Christoph Luitpold, " La villa e i giardini del Quirinale nel Cinquecento ", Bollettino d'Arte, nº́cial (Restauri al Quirinale), 1999, p. 15-62.

Frommel Christoph Luitpold, "Il "ninfeo" die Bramante a Genazzano », dans Frommel, C. L., Architettura alla corte papale nel Rinascimento, Milan, 2003a, p. 215-239.

Frommel Christoph Luitpold (dir.), La Villa Farnesina à Rome, Modène, Mirabilia Italiæ, 2003 b.

Frommel Sabine, Sebastiano Serlio architecte (éd. fr. de Sebastiano Serlio architetto, Milan, 1998), Paris, 2002a.

Frommel Sabine, "Sogni architettonici: i Sangallo e la tipologia di palazzi e ville a pianta centralizzata ", Quaderni dell'Istituto di Storia dell'Architettura, n ${ }^{\circ} 40$, 2002b, p. 17-38.

Frommel Sabine, "Premières expériences : entre sculpture, construction et poésie. La grotte des Pins, la fontaine d'Hercule, l'architecture imaginée ", dans Frommel Sabine (dir.), Primatice architecte, Picard, Paris, 2010a, p. 87-112.

Frommel Sabine, "Vers l'architecture monumentale : les travaux pour Charles de Guise (1550-1559) ", dans Frommel Sabine (dir.), Primatice architecte, Paris, Picard, 2010b, p. 113-149.

Frommel Sabine, "Deux couples et deux stratégies : Marguerite d'Autriche et Octave Farnèse, Catherine de Médicis et Henri II ", dans Frommel Sabine et Dumas Juliette, Bâtir au féminin? Traditions et stratégies en Europe et dans l'Empire ottoman, (coll. itinéraires/percorsi 2), Picard, Paris, 2013, p. 153-170.

Frommel Sabine, "Sebastiano Serlio's padiglione al costume di Franza in Fontainebleau und sein Beitrag für die Entwicklung der Badekultur am französischen Hof ", dans Deutsch Kristina, Echinger-Maurach Claudia et 
Krems Eva-Bettina, Höfische Bäder in der frühen Neuzeit, Gestalt und Funktion, De Gruyter, Berlin/Boston, 2017, p. 66-88.

Frommel Sabine, Leuschner Eckhard, Droguet Vincent et al, Construire avec le corps humain. Bauen mit dem menschlichen Körper, 2 vol., Campisano Editore, Rome, 2018.

LuRIN Emmanuel, " "Robbe di fontane per la Francia" : Les matériaux de rocaille envoyés par Ferdinand Ier de Médicis à Henri IV ", dans Ferretti, Frommel, Giannotti, Mozzo (dir.), « Il Rinascimento delle grotte. Natura, arte e architettura fra Italia e Francia nel Cinquecento ", Opus Incertum, Nuova Serie, anno IV, 2018, p. 112-125.

Magnani Lauro, Tra magia, scienze e "meraviglia": le grotte artificiali dei giardini genovesi nei secoli XVI e XVII, Sagep, Gênes, 1984.

Modesti Paola, Le Delizie ritrovate. Poggioreale e la villa del Rinascimento nella Napoli aragonese, Olschki, Florence, 2014.

Morel Philippe, Les Grottes maniéristes en Italie au XVt siècle : théâtre et alchimie de la nature, Macula, Paris, 1998.

Pоснат Götz, Theater und bildende Künste. Im Mittelalter und in der Renaissance in Italien, Adeva, Graz, 1990.

Romana Liserre Francesca, Grotte e ninfei nel '500. Il modello dei giardini di Caprarola, Gangemi, Roma, 2008.

Taglialagamba Sara, "Architectures théâtrales, fêtes et constructions éphémères chez Léonard ", dans Frommel Sabine et Guillaume Jean, Léonard de Vinci et l'architecture, Mare \& Martin, Paris, 2019, p. 87-97.

\section{L’autrice}

Depuis 2003, Sabine Frommel est directrice d'études à la direction d'études Histoire de l'Art de la Renaissance à l'École pratique des hautes études (Sorbonne, PSL). De 2013 à 2015, elle a été professeur invité à l'université de Bologne (Dipartimento di Filologia classica e Italianistica). Ses recherches couvrent un vaste éventail de thématiques transversales : l'évolution des typologies et des langages architecturaux au Quattrocento et Cinquecento ; les processus de migration de modèles et de techniques en Europe ; la représentation de l'architecture dans la peinture ; l'héritage de la Renaissance jusqu'au xIx ${ }^{e}$ siècle. 